\title{
Small Solutions for Big Problems: The Application of Nanoparticles to Brain Tumor Diagnosis and Therapy
}

DA Orringer ${ }^{1}$, YE Koo ${ }^{2}$, T Chen ${ }^{3}$, R Kopelman ${ }^{2}$, O Sagher ${ }^{1}$ and MA Philbert ${ }^{4}$

Nanotechnology has been projected to have a significant impact on the future treatment of brain tumors. Specifically, nanoparticles have the potential to revolutionize brain tumor imaging as well as surgical and adjuvant treatments. The translation of current research in nanotechnology into clinical practice will rely on solving challenges relating to the pharmacology of nanoparticles.

\section{WHY NANOPARTICLES?}

One of the key reasons that nanoparticles have promise in the treatment of cancer is that they can be targeted to tumors through antigen-dependent (specific) or antigen-independent (nonspecific) mechanisms. Specific targeting relies on the interaction of antigens on the surface of nanoparticles with tumor cell receptors. A variety of molecules including peptides (arginineglycine-aspartic acid, ${ }^{1} \mathrm{~F} 3,{ }^{2}$ and chlorotoxin ${ }^{3}$ ), cytokines (interleukin-13 $3^{4}$, drugs (methotrexate ${ }^{5}$ ), antibodies (anti-epithelial growth factor antibodies ${ }^{6}$ ), and ferromagnetic agents ${ }^{7}$ have been proposed as targeting modalities. Multiple targeting molecules can be added to the surface of nanoparticles to tailor targeting of brain tumors through a concept referred to as "surface-mediated multivalent affinity effects." 1

Nonspecific targeting relies on the preferential extravasation of nanoparticles into the brain through vascular access provided by blood-brain barrier (BBB) breakdown, which occurs in many brain tumors. Other small molecules can also cross BBB defects. However, unlike small molecules that diffuse freely into and out of a tumor, nanoparticles accumulate within a tumor because of the enhanced permeability and retention effect. The effect accounts for the observation that nanoparticles are retained within tumor tissue after serum levels decline. The enhanced permeability and retention effect results from active angiogenesis, the expression of vascular mediators of extravasation, and altered vascular architecture. ${ }^{8}$
In addition to their potential for targeting, the physicochemical properties of nanoparticles make them ideal devices for the delivery of compounds to brain tumors. Molecules such as contrast agents or drugs can be loaded into the core of a nanoparticle or applied as a coating to its surface. The process of a single nanoparticle carrying a large number of drug molecules or ions is referred to as "nanoparticle amplification" ${ }^{\text {and explains the }}$ concept of nanoparticles as delivery devices. In addition, molecules with different functions can be incorporated into a nanoparticle to create multifunctional nanoparticles (Figure 1). The size and chemical composition of a nanoparticle can be altered to control the efficiency of small-molecule loading.

The performance of nanoparticles in biological systems suggests that by isolating their payload from the surrounding environment, they may reduce the systemic toxicity associated with conventional chemotherapeutic agents. Moreover, nanoparticles create a barrier to degradation of their payload by preventing contact with plasma enzymes.

\section{DIAGNOSTIC APPLICATIONS IO MRI contrast agents}

One of the most mature applications of nanotechnology to the diagnosis of brain tumors is in magnetic resonance imaging (MRI). Various nanoparticles have been developed as MRI contrast agents. To date, nanoparticle-based contrast agents have been designed with a core of iron oxide (IO) crystals with or without a shell of organic material, such as polyethylene glycol (PEG). ${ }^{9-11}$

The key benefit of nanoparticle-based materials is that they may provide better information about the extent of tumor. Both gadolinium-based contrast agents and nanoparticle-based contrast agents cause enhancement of tumors by passing through areas of disrupted BBB where they alter MR signal intensity. However, unlike gadolinium, nanoparticle-based contrast agents,

\footnotetext{
'Department of Neurosurgery, University of Michigan Medical School, Ann Arbor, Michigan, USA; ${ }^{2}$ Department of Chemistry, University of Michigan, Ann Arbor, Michigan, USA; ${ }^{3}$ University of Michigan, Ann Arbor, Michigan, USA; ${ }^{4}$ Department of Environmental Health Sciences, University of Michigan, Ann Arbor, Michigan, USA. Correspondence: MA Philbert (philbert@umich.edu)
} 
such as ultrasmall superparamagnetic IO (SPIO) nanoparticles, are taken up by the phagocytes found at tumor margins. ${ }^{12}$ Therefore, areas of tumor not seen with gadolinium-enhanced MRI can be detected using IO-based nanoparticles. ${ }^{11}$ Moreover, unlike freely diffusing gadolinium chelates, IO-based nanoparticles tend to persist longer within the tumor and more accurately delineate tumor margins. ${ }^{12}$ Another advantage of nanoparticles is their capacity for highly selective molecular tumor targeting. It is possible that nanoparticles could be engineered to image certain subpopulations of cells, such as stem cells or endothelial cells. Various IO nanoparticles under development show promise as tumor-specific contrast agents. ${ }^{2,13,14}$

\section{Intraoperative brain tumor delineation}

The central challenge of brain tumor surgery is achieving a complete resection without damaging normal structures near the tumor. Achieving maximal resection currently relies on the neurosurgeon's ability to judge the presence of residual tumor during surgery. The use of fluorescent and visible dyes has been proposed as a means of visualizing tumor margins intraoperatively. However, investigators have been hampered by three main difficulties: (i) achieving tumor specificity, (ii) achieving adequate visual contrast, and (iii) identifying a dye useful for a wide range of tumors. Dye-loaded nanoparticles have been reported to meet each of these challenges.

IO-based nanoparticles loaded with the near-infrared fluorescing molecule Cy5.5 for intraoperative tumor delineation have been created and characterized. ${ }^{13,14}$ These nanoparticles can be visualized in experimental models with fluorescence imaging and MRI. Under the appropriate lighting conditions, Cy5.5-loaded nanoparticles delineate margins of implanted tumors (Figure 2). ${ }^{15}$

Optical semiconductor nanocrystals, called quantum dots, have also been evaluated as a method to visualize brain tumors

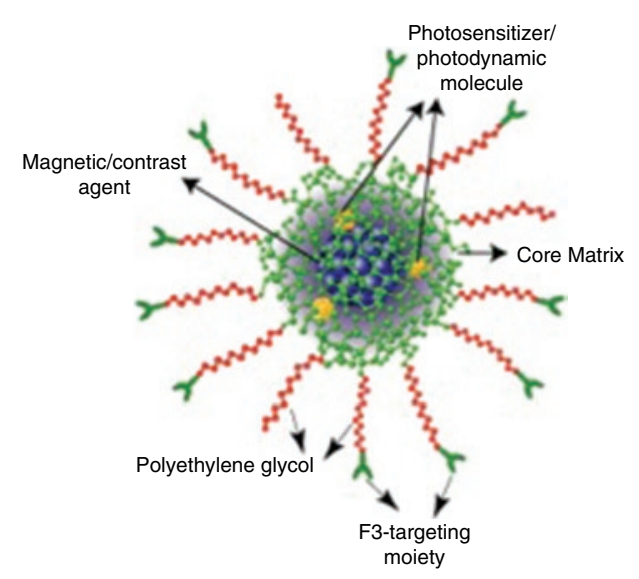

Figure 1 Schematic diagram of multifunctional polyacrylamide nanoparticle. While this nanoparticle contains contrast agents, photosensitizers, and F3-targeting peptides, all of these components can be interchanged for other small molecules with various functions. The size of the nanoparticle shown is $\sim 20 \mathrm{~nm}$. Generally larger than circulating proteins (hemoglobin tetramer $(6 \mathrm{~nm})$ and albumin $(8 \mathrm{~nm})$ ), nanoparticles range in size from 1 to $100 \mathrm{~nm}$. Adapted with permission from Reddy et al. Vascular targeted nanoparticles for imaging and treatment of brain tumors. Clin Cancer Res 12: 6677-6686 (2006). Figure 1. and have been shown to induce fluorescent staining of implanted C6 brain tumors. ${ }^{16}$ However, because of their heavy metal content, quantum dots are potentially toxic toward normal tissues. Moreover, like Cy5.5-loaded nanoparticles, the quantum dots characterized to date require a darkened operative field for visualization. Still, it is possible that the composition of the quantum dots could be modified to emit light in the visible spectrum. ${ }^{16}$

We have developed and characterized a nontoxic dye-loaded polyacrylamide nanoparticle that shows promise for delineating neoplastic tissue under normal lighting conditions. Coomassie-blue loaded nanoparticles have been shown to visibly stain 9L gliosarcoma cells, and in vivo data are currently being collected.

\section{THERAPEUTIC APPLICATIONS Chemotherapeutics}

Nanoparticles are in a unique position to enable the development of novel chemotherapeutics by facilitating passage of these compounds across the $\mathrm{BBB}$ or across the blood-tumor barrier and delivering drugs to brain tumors at levels that would not otherwise be possible. Nanoparticles may also meet the challenge of efficiently delivering hydrophobic drugs to tumor cells and overcoming drug resistance.

Solid lipid nanoparticles (SLNs) are among the best characterized, nontoxic, nanoscale devices for brain tumor drug delivery. Although the exact mechanism by which SLNs cross the BBB and blood-tumor barrier is unknown, binding, endocytosis, and phagocytosis by endothelial cells are central components. ${ }^{17}$ The lipid matrix of SLN provides a means of loading drugs such as doxorubicin and paclitaxel in a microenvironment that protects them from degradation and improves their therapeutic window by maximizing release within tumor tissue. Drug-loaded SLNs have been shown to enhance tumor concentrations and decrease plasma concentrations of doxorubicin and paclitaxel as compared to equivalent drug doses, even without the need for toxic surfactants. ${ }^{17}$ The unloading of drugs within target tumor tissues can also be controlled through modification of the SLN surface and constituent lipids. ${ }^{18}$

Similar in composition to SLNs, nanoparticle formulations of low-density lipoproteins have also been proposed as novel drug delivery devices. Tumor cells preferentially take up low-density lipoprotein nanoparticles via corresponding receptors, which are
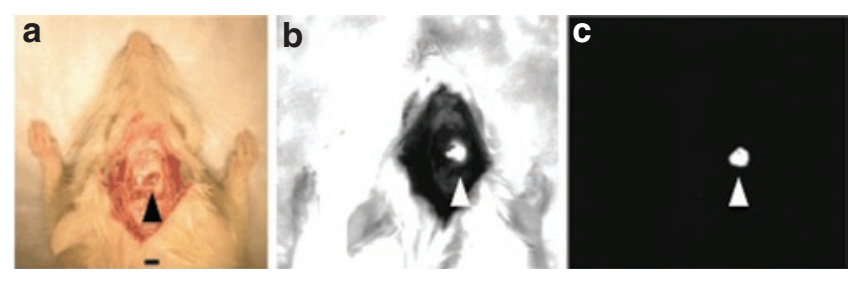

Figure 2 Fluorescent staining of implanted green fluorescent protein (GFP)expressing $9 \mathrm{~L}$ gliosarcoma by nanoparticles containing Cy5.5 (near-infrared dye). The tumor is visualized under (a) normal lighting conditions, (b) GFP channel, and (c) Cy5.5 channel. The tumor is above the black or white triangle. Adapted with permission from Kircher MF, Mahmood U, King RS, Weissleder R, Josephson L. A multimodal nanoparticle for preoperative magnetic resonance imaging and intraoperative optical brain tumor delineation. Cancer Res 63: 8122-8125. 
upregulated in these tissues. The utility of low-density lipoprotein nanoparticles as drug delivery devices has been suggested by in vitro studies that demonstrate their rapid internalization by glioma cell lines. ${ }^{19}$

Like lipid-based nanoparticles, non-lipid-based nanoparticles consisting of matrices of synthetic biocompatible polymers also isolate their payload from the environment. The clinical use of doxorubicin ${ }^{20}$ and paclitaxel ${ }^{21}$ in gliomas has been limited by the inability of these drugs to cross the BBB because of the p-glycoprotein drug efflux system in endothelial cells. The feasibility of employing polymeric doxorubicin-loaded nanoparticles for the treatment of brain tumors has been demonstrated in a rat glioma model. ${ }^{20}$ Similarly, paclitaxel-loaded poly(D,L-lactide-co-glycolide) nanoparticles were more cytotoxic to $\mathrm{C} 6$ glioma cells than was free paclitaxel in vitro, probably due to internalization and intracellular unloading of the drug from the nanoparticles. ${ }^{22}$ Moreover, when paclitaxel is used, poly(D,L-lactide-co-glycolide) nanoparticles eliminate the need for coadministration of surfactant. Data from clinical trials of Abraxane, a nanoparticle formulation of paclitaxel, in cancer patients will help to determine how nanoparticle-based drugs may be applied in treating glioma patients.

In addition to enabling the entry of chemotherapeutics into tumor cells, nanoparticles can be engineered to potentiate the activity of chemotherapeutics by inhibiting the p-glycoprotein drug efflux system, which confers resistance to therapy to some tumor cells. Specifically, polycyanoacrylate, cetyl alcohol/ polysorbate, lipid polymer-based, and surfactant polymerbased nanoparticles have been shown to potentiate the effects of chemotherapeutic agents by inhibiting p-glycoprotein activity in glioma cell lines. ${ }^{23}$ The activity of chemotherapeutic compounds in glioma cells has also been potentiated when they are co-incorporated with nanoparticles containing p-glycoprotein inhibitors. $^{24}$

\section{Nonconventional therapeutics}

In addition to the delivery of conventional anticancer agents, nanoparticles may be capable of delivering gene therapy plasmids. Nanoparticles are gaining favor as vectors for gene therapy because they cross the BBB more efficiently, can be dosed more precisely, and are less immunogenic than traditional vectors. Recently, a plasmid encoding proapoptotic Apo2 ligand/tumor necrosis factor-related apoptosis-inducing ligand was incorporated into a cationic albumin-conjugated PEGylated nanoparticle and found to have potent antitumor activity against the C6 glioma model in vitro and in vivo. ${ }^{25}$

Attempts at thermotherapy for the treatment of brain tumors using focused ultrasound have been hampered by the challenges of the electromagnetic properties of the skull and of achieving even temperature distribution throughout a lesion. ${ }^{26}$ An alternative strategy was developed in which SPIO nanoparticles were injected intratumorally and heated via a magnetic field. SPIO nanoparticles are well suited for this application since they are retained within tumor tissue for the duration of therapy. Injected SPIO nanoparticles were first tested in a rat model of glioma and shown to be effective in improving survival. ${ }^{27}$ A recent phase I clinical trial suggests that SPIO can be safely administered to patients with glioblastoma. ${ }^{26}$

Photodynamic therapy (PDT) is an experimental adjuvant therapy for brain tumors that carries little local or systemic treatment associated morbidity. PDT was initially applied clinically to cutaneous and bladder malignancies, which can easily be exposed to light. While brain tumors cannot be exposed to light as easily, even the deepest brain tumors become exposed during surgery. Thus far, the efficacy of PDT for treating brain tumors has been limited in clinical trials, probably because of the difficulty of creating tumor-specific, sufficient accumulation of photosensitizer within neoplastic cells. ${ }^{28}$ Polymeric nanoparticles offer a solution to this problem by allowing the delivery of a large quantity of photosensitizers to tumor cells via tumorspecific ligands. Reddy et al. have induced long-term remission of implanted 9L gliomas through PDT mediated by F3-targeted, Photofrin-loaded magnetic nanoparticles. ${ }^{2}$ The ability of nanoparticles to mediate PDT is an exciting possibility that merits further investigation.

\section{FUTURE CHALLENGES}

Challenges related to nanoparticle clearance and toxicity must be overcome before nanoparticles can be used clinically. While the addition of PEG to the nanoparticle surface can prevent opsonization and delay clearance, ${ }^{29}$ the clearance of PEGylated nanoparticles through the liver is relatively slow, thereby increasing the risk of toxicity. Because of conflicting evidence in various animal models, lack of details about the mechanism by which PEG prevents opsonization, and a limited understanding of the biochemical properties of PEG polymers for coating nanoparticles, significant experimental work remains to be done before PEGylated nanoparticles can be used clinically. ${ }^{30}$

Beyond preliminary evidence that suggests that nanoparticles may have toxicity toward astrocytes and neurons in culture, there are few in vitro data on the toxicity of nanoparticles toward the central nervous system. Nonetheless, nanoparticles have been used in experimental studies in humans as imaging and drug delivery agents without significant adverse consequences. An understanding of the relationship between toxicity and particle size, geometry, pharmacokinetics, and surface coating is required before nanoparticles can be used in clinical practice.

\section{SUMMARY}

Nanoparticles have the potential to advance the diagnosis, operative management, and adjuvant therapy of brain tumors. Nanoparticle-based MR contrast agents have the potential to visualize portions of tumor, especially along the tumor-brain interface, that would have been unclear with conventional MRI. In addition, nanoparticles may ultimately improve the completeness of brain tumor resection. Finally, delivery of chemotherapy and nontraditional therapies to brain tumors will likely be improved by nanoparticle-based drug delivery devices. As our knowledge of their pharmacology expands, nanoparticles are likely to play a central role in the future management of brain tumor patients. 


\section{ACKNOWLEDGMENTS}

The authors thank John Cowan Jr. for his assistance in the preparation of this article. This work was supported by grants 1R01EB007977-01 (R.K.), 1R21CA125297-01A1 (R.K.), and 1F32CA126295-01A1 (D.A.O.) and by a CNS Basic/Translational Resident Research Fellowship (D.A.O.)

\section{CONFLICT OF INTEREST}

The authors declared no conflict of interest.

๑) 2009 American Society for Clinical Pharmacology and Therapeutics

1. Montet, X., Funovics, M., Montet-Abou, K., Weissleder, R. \& Josephson, L. Multivalent effects of RGD peptides obtained by nanoparticle display. J. Med. Chem. 49, 6087-6093 (2006).

2. Reddy, G.R. et al. Vascular targeted nanoparticles for imaging and treatment of brain tumors. Clin. Cancer Res. 12, 6677-6686 (2006).

3. Sun, C. et al. In vivo MRI detection of gliomas by chlorotoxin-conjugated superparamagnetic nanoprobes. Small 4, 372-379 (2008).

4. Madhankumar, A.B., Slagle-Webb, B., Mintz, A., Sheehan, J.M. \& Connor, J.R. Interleukin-13 receptor-targeted nanovesicles are a potential therapy for glioblastoma multiforme. Mol. Cancer Ther. 5, 3162-3169 (2006).

5. Dhanikula, R.S., Argaw, A., Bouchard, J.F. \& Hildgen, P. Methotrexate loaded polyether-copolyester dendrimers for the treatment of gliomas: enhanced efficacy and intratumoral transport capability. Mol. Pharm. 5, 105-116 (2008).

6. Tsutsui, Y. et al. Development of bionanocapsules targeting brain tumors. J. Control. Release 122, 159-164 (2007).

7. Chertok, B. et al. Iron oxide nanoparticles as a drug delivery vehicle for MR monitored magnetic targeting of brain tumors. Biomaterials 29, 487-496 (2008).

8. Maeda, H. The enhanced permeability and retention (EPR) effect in tumor vasculature: the key role of tumor-selective macromolecular drug targeting Adv. Enzyme Regul. 41, 189-207 (2001).

9. Moffat, B.A. et al. A novel polyacrylamide magnetic nanoparticle contrast agent for molecular imaging using MRI. Mol. Imaging 2, 324-332 (2003).

10. Peira, E., Marzola, P., Podio, V., Aime, S., Sbarbati, A. \& Gasco, M.R. In vitro and in vivo study of solid lipid nanoparticles loaded with superparamagnetic iron oxide. J. Drug Target. 11, 19-24 (2003).

11. Neuwelt, E.A. et al. The potential of ferumoxytol nanoparticle magnetic resonance imaging, perfusion, and angiography in central nervous system malignancy: a pilot study. Neurosurgery 60, 601-611; discussion 611-612 (2007).

12. Varallyay, P. et al. Comparison of two superparamagnetic viral-sized iron oxide particles ferumoxides and ferumoxtran-10 with a gadolinium chelate in imaging intracranial tumors. AJNR Am. J. Neuroradiol. 23, 510-519 (2002).

13. Kircher, M.F., Mahmood, U., King, R.S., Weissleder, R. \& Josephson, L. A multimodal nanoparticle for preoperative magnetic resonance imaging and intraoperative optical brain tumor delineation. Cancer Res. 63, 8122-8125 (2003).

14. Veiseh, O. et al. Optical and MRI multifunctional nanoprobe for targeting gliomas. Nano Lett. 5, 1003-1008 (2005).
15. Trehin, R., Figueiredo, J.L., Pittet, M.J., Weissleder, R., Josephson, L. \& Mahmood, U. Fluorescent nanoparticle uptake for brain tumor visualization. Neoplasia 8, 302-311 (2006).

16. Jackson, H. et al. Quantum dots are phagocytized by macrophages and colocalize with experimental gliomas. Neurosurgery 60, 524-529; discussion 529-530 (2007).

17. Brioschi, A., Zenga, F., Zara, G.P., Gasco, M.R., Ducati, A.\& Mauro, A. Solid lipid nanoparticles: could they help to improve the efficacy of pharmacologic treatments for brain tumors? Neurol. Res. 29, 324-330 (2007).

18. Zara, G.P., Cavalli, R., Bargoni, A., Fundarò, A., Vighetto, D. \& Gasco, M.R. Intravenous administration to rabbits of non-stealth and stealth doxorubicinloaded solid lipid nanoparticles at increasing concentrations of stealth agent: pharmacokinetics and distribution of doxorubicin in brain and other tissues. J. Drug Target. 10, 327-335 (2002).

19. Nikanjam, M., Gibbs, A.R., Hunt, C.A., Budinger, T.F. \& Forte, T.M. Synthetic nano-LDL with paclitaxel oleate as a targeted drug delivery vehicle for glioblastoma multiforme. J. Control. Release 124, 163-171 (2007).

20. Steiniger, S.C. et al. Chemotherapy of glioblastoma in rats using doxorubicinloaded nanoparticles. Int. J. Cancer 109, 759-767 (2004).

21. Cahan, M.A., Walter, K.A., Colvin, O.M. \& Brem, H. Cytotoxicity of taxol in vitro against human and rat malignant brain tumors. Cancer Chemother. Pharmacol. 33, 441-444 (1994).

22. Dong, Y. \& Feng, S.S. Poly(D,L-lactide-co-glycolide) (PLGA) nanoparticles prepared by high pressure homogenization for paclitaxel chemotherapy. Int. J. Pharm. 342, 208-214 (2007).

23. Chavanpatil, M.D. et al. Surfactant-polymer nanoparticles overcome P-glycoprotein-mediated drug efflux. Mol. Pharm. 4, 730-738 (2007).

24. Soma, C.E., Dubernet, C., Bentolila, D., Benita, S. \& Couvreur, P. Reversion of multidrug resistance by co-encapsulation of doxorubicin and cyclosporin A in polyalkylcyanoacrylate nanoparticles. Biomaterials 21, 1-7 (2000).

25. Lu, W., Sun, Q., Wan, J., She, Z. \& Jiang, X.G. Cationic albumin-conjugated pegylated nanoparticles allow gene delivery into brain tumors via intravenous administration. Cancer Res. 66, 11878-11887 (2006).

26. Maier-Hauff, K. et al. Intracranial thermotherapy using magnetic nanoparticles combined with external beam radiotherapy: results of a feasibility study on patients with glioblastoma multiforme. J. Neurooncol. 81, 53-60 (2007).

27. Jordan, A. et al. The effect of thermotherapy using magnetic nanoparticles on rat malignant glioma. J. Neurooncol. 78, 7-14 (2006).

28. Stylli, S.S., Howes, M., MacGregor, L., Rajendra, P. \& Kaye, A.H. Photodynamic therapy of brain tumours: evaluation of porphyrin uptake versus clinical outcome. J. Clin. Neurosci. 11, 584-596 (2004).

29. Vijayaraghavalu, S., Raghavan, D. \& Labhasetwar, V. Nanoparticles for delivery of chemotherapeutic agents to tumors. Curr. Opin. Investig. Drugs 8, 477-484 (2007)

30. Owens, D.E. 3rd. \& Peppas, N.A. Opsonization, biodistribution, and pharmacokinetics of polymeric nanoparticles. Int. J. Pharm. 307, 93-102 (2006). 